\title{
ON NONCOLLAPSED ALMOST RICCI-FLAT 4-MANIFOLDS
}

\author{
VITALI KAPOVITCH AND JOHN LOTT
}

\begin{abstract}
We give topological conditions to ensure that a noncollapsed almost Ricci-flat 4-manifold admits a Ricci-flat metric. One sufficient condition is that the manifold is spin and has a nonzero $\hat{A}$-genus. Another condition is that the fundamental group is infinite or, more generally, of sufficiently large cardinality.
\end{abstract}

\section{INTRODUCTION}

One of the most basic pinching theorems in Riemannian geometry says that a noncollapsed almost flat manifold admits a flat metric. The word noncollapsed refers to a lower volume bound. More precisely, given $n \in \mathbb{Z}^{+}$and $v>0$, there is some $\epsilon=\epsilon(n, v)>0$ so that if $(M, g)$ is a Riemannian $n$-manifold with $\operatorname{vol}(M) \geq v, \operatorname{diam}(M) \leq 1$ and $\left|\mathrm{Rm}_{M}\right| \leq \epsilon$, then $M$ admits a flat Riemannian metric. Here $\mathrm{Rm}_{M}$ denotes the Riemann curvature tensor. This result seems to have first been stated by Gromov in Gr78, where he noted that it follows from Cheeger's arguments in Ch69. (The point of [Gr78] was to characterize what happens when one removes the volume assumption.)

One can ask if there is an analogous statement for noncollapsed almost Ricci-flat manifolds. In dimension less than four, being almost Ricci-flat is the same as being almost flat. Hence the first interesting case is in dimension four. We give topological conditions to ensure that a noncollapsed almost Ricci-flat 4-manifold admits a Ricci-flat metric. We also give more general results about noncollapsed manifolds with almost nonnegative Ricci curvature, or almost nonnegative scalar curvature and bounded Ricci curvature.

In the rest of the introduction we state the main results, outline the proof of Theorem 1.1, mention some earlier related results and give the structure of the paper.

Date: October 13, 2017.

The first author was partially supported by an NSERC Discovery Grant. The second author was partially supported by NSF grant DMS-1510192. Research at MSRI was partially supported by NSF grant DMS-1440140. We thank MSRI for its hospitality during the Spring 2016 program. 
1.1. Statement of results. The first result is in four dimensions. Recall that the $\widehat{A}$-genus $\widehat{A}(M)$ of a closed oriented manifold $M$ is a certain rational combination of the Pontryagin numbers of $M$. In four dimensions, $\widehat{A}(M)$ equals minus one eighth of the signature of $M$. We consider a noncollapsed spin 4-manifold with almost nonnegative scalar curvature and a nonzero $\widehat{A}$-genus. With an upper bound on the Ricci curvature, the conclusion is that the manifold must be diffeomorphic to a $K 3$ surface.

Let $S$ denote scalar curvature.

Theorem 1.1. Given $v>0$ and $\Upsilon<\infty$, there is an $\varepsilon=\varepsilon(v, \Upsilon)>$ 0 with the following property. Suppose that $(M, g)$ is a closed connected Riemannian spin 4-manifold with $\hat{A}(M) \neq 0, \operatorname{vol}(M) \geq v$, $\operatorname{diam}(M) \leq 1$, Ric $_{M} \leq \Upsilon \mathrm{g}$ and $S_{M} \geq-\epsilon$. Then $M$ is diffeomorphic to a K3 surface.

As a consequence, if a noncollapsed almost Ricci-flat 4-manifold is spin, and has a nonzero $\widehat{A}$-genus, then it admits a Ricci-flat metric.

Corollary 1.2. Given $v>0$, there is an $\varepsilon^{\prime}=\varepsilon^{\prime}(v)>0$ with the following property. Suppose that $(M, g)$ is a closed connected Riemannian spin 4-manifold with $\hat{A}(M) \neq 0, \operatorname{vol}(M) \geq v, \operatorname{diam}(M) \leq 1$ and $\mid$ Ric $_{M} \mid \leq \varepsilon^{\prime}$. Then $M$ is diffeomorphic to a K3 surface. In particular, $M$ admits a Ricci-flat metric.

For example, if $M=K 3 \#\left(S^{2} \times S^{2}\right)$ then there is no Riemannian metric on $M$ with $\operatorname{vol}(M) \geq v, \operatorname{diam}(M) \leq 1$ and $\left|\operatorname{Ric}_{M}\right| \leq \varepsilon^{\prime}$.

If $\pi_{1}(M)$ is infinite, or of sufficiently large cardinality, then we have the following related $n$-dimensional result, which does not involve any spin assumption on $M$.

Theorem 1.3. Given $n \in \mathbb{Z}^{+}, v>0$ and $\Lambda, \Upsilon<\infty$, there exist $\varepsilon=\varepsilon(n, v, \Lambda, \Upsilon)>0, C=C(n, v, \Lambda, \Upsilon)<\infty, \varepsilon^{\prime}=\varepsilon^{\prime}(n, v, \Lambda)>0$ and $C^{\prime}=C^{\prime}(n, v, \Lambda)<\infty$ with the following property. Suppose that $(M, g)$ is an $n$-dimensional closed connected Riemannian manifold with $\int_{M}\left|R m_{M}\right|^{\frac{n}{2}} d v o l_{M} \leq \Lambda, \operatorname{vol}(M) \geq v$ and $\operatorname{diam}(M) \leq 1$.

(1) If $-\epsilon g \leq R i c_{M} \leq \Upsilon g$ and $\left|\pi_{1}(M)\right| \geq C$ then $M$ admits a $W^{2, p}$-regular Riemannian metric $h$ with nonnegative measurable Ricci curvature for which the universal cover $(\tilde{M}, \tilde{h})$ isometrically splits off an $\mathbb{R}$-factor. In particular, $\pi_{1}(M)$ is infinite.

(2) If $\left|R i c_{M}\right| \leq \varepsilon^{\prime}$ and $\left|\pi_{1}(M)\right| \geq C^{\prime}$ then $M$ admits a Ricci-flat metric $h^{\prime}$ for which the universal cover $\left(\tilde{M}, \tilde{h}^{\prime}\right)$ isometrically splits off an $\mathbb{R}$-factor. 
In the four dimensional case, the conclusions of Theorem 1.3 can be made more precise. Theorem 1.3)(1) becomes a statement about manifolds with almost nonnegative Ricci curvature, again under an upper Ricci curvature bound. Theorem [1.3(2) becomes a second sufficient topological condition, involving the fundamental group, for a noncollapsed almost Ricci-flat 4-manifold to admit a Ricci-flat metric.

Corollary 1.4. Given $v>0$ and $\Upsilon<\infty$, there exist $\varepsilon^{\prime \prime}=\varepsilon^{\prime \prime}(v, \Upsilon)>$ $0, C^{\prime \prime}=C^{\prime \prime}(v, \Upsilon)<\infty, \varepsilon^{\prime \prime \prime}=\varepsilon^{\prime \prime \prime}(v)>0$ and $C^{\prime \prime \prime}=C^{\prime \prime \prime}(v)<\infty$ with the following property. Suppose that $(M, g)$ is a closed connected Riemannian 4-manifold with $\operatorname{vol}(M) \geq v$ and $\operatorname{diam}(M) \leq 1$.

(1) If $-\varepsilon^{\prime \prime} g \leq R i c_{M} \leq \Upsilon g$ and $\left|\pi_{1}(M)\right| \geq C^{\prime \prime}$ then $M$ admits a smooth Riemannian metric with nonnegative Ricci curvature for which the universal cover $(\tilde{M}, \tilde{g})$ isometrically splits off an $\mathbb{R}$-factor.

(2) If $\mid$ Ric $_{M} \mid \leq \varepsilon^{\prime \prime \prime}$ and $\left|\pi_{1}(M)\right| \geq C^{\prime \prime \prime}$ then $M$ admits a flat metric.

As an example of Corollary 1.4(2), let $M$ be the result of performing surgery along an embedded circle in $T^{4}$, i.e. removing a tubular neighborhood of the circle and attaching a copy of $D^{2} \times S^{2}$. Then there is no Riemannian metric on $M$ with $\operatorname{vol}(M) \geq v, \operatorname{diam}(M) \leq 1$ and $\left|\operatorname{Ric}_{M}\right| \leq \varepsilon^{\prime \prime \prime}$. (On the other hand, Anderson showed that if the circle is a meridian curve in $T^{4}$, and one performs surgery with respect to the canonical trivialization of its normal bundle, then the resulting manifold does admit a sequence of collapsing almost Ricci-flat metrics An92, Theorem 0.4].)

Remark 1.5. The diffeomorphism types of the manifolds $M$ in the conclusion of Corollary 1.4(1) are easy to describe, using [Ha86, Section 9].

Remark 1.6. The known Ricci-flat closed 4-manifolds are flat (hence with infinite fundamental group) or are finitely covered by a $K 3$ surface (which is spin and has $\widehat{A}(K 3)=2$ ). In view of this fact, the topological hypotheses of Corollary 1.2 and Corollary 1.4(2) are not unreasonable.

Finally, we give a fundamental group restriction on noncollapsed manifolds with almost nonnegative Ricci curvature.

Theorem 1.7. Given $n \in \mathbb{Z}^{+}$and $v>0$, there are $\varepsilon=\varepsilon(n, v)>$ 0 and $I=I(n, v)<\infty$ with the following property. Suppose that $(M, g)$ is a closed connected Riemannian $n$-manifold with $\operatorname{vol}(M) \geq v$, $\operatorname{diam}(M) \leq 1$ and Ric $_{M} \geq-\varepsilon$. Then $\pi_{1}(M)$ has an abelian subgroup (of index at most I) generated by at most $n$ elements. 
1.2. Outline of the proof of Theorem 1.1. If the theorem fails then we take a sequence of counterexamples, with $\epsilon \rightarrow 0$. The hypotheses imply that there is a uniform lower Ricci curvature bound. From a result of Cheeger and Naber, there is an a priori upper bound on $\int_{M}\left|\mathrm{Rm}_{M}\right|^{2} \mathrm{dvol}_{M}$ CN15]. The first part of the argument for Theorem 1.1 is now standard, based on ideas of Anderson An90, AndersonCheeger AC91 and Bando Ba90a. We can pass to a subsequence that converges, in the Gromov-Hausdorff topology, to a four dimensional orbifold $X$ that has a finite number of orbifold singular points, and a $C^{1, \alpha}$-regular Riemannian metric $g_{X}$ on its regular part $X_{\text {reg }}$. Doing appropriate blowups to zoom in on the formation of singular points of $X$, one obtains noncompact Ricci-flat ALE orbifolds. Doing further blowups to zoom in on the formation of their singular points, one obtains a bubble tree whose vertices correspond to Ricci-flat orbifolds. By assembling the geometric pieces in the bubble tree, one can reconstruct the diffeomorphism type of the original manifold.

In order to proceed, we need more information about $X$ and the ALE blowups. The assumption that $M$ is spin, with nonzero $\widehat{A}$-genus, helps in several ways. First, the nonvanishing of the $\widehat{A}$-genus of $M$ implies that $M$ has a nonvanishing harmonic spinor field. We show that these harmonic spinor fields pass to a nonzero parallel spinor field on $X_{\text {reg }}$. This forces $X_{\text {reg }}$ to be smooth and Ricci-flat. We show that $X$ is a Ricci-flat spin orbifold. The existence of the parallel spinor field now implies that $X$ is a hyperKähler orbifold.

The spin assumption on $M$ also implies that the Ricci-flat ALE blowup orbifolds are spin. Compatibility of spin structures, along with the existence of the nonzero parallel spinor field on $X$, means that the blowup orbifolds have spinor fields that are asymptotically parallel at infinity, and nonzero there. Then a small variation on a result of Nakajima [Na90, says that the blowup orbifolds are hyperKähler. One knows enough about hyperKähler ALE 4-manifolds, and 4-orbifolds, to conclude that after assembling the pieces in the bubble tree, the result is diffeomorphic to a compact hyperKähler manifold. Theorem 1.1 follows from this fact.

1.3. Related results. Anderson gave a Ricci pinching result for Riemannian metrics on $S^{4}$ and $\mathbb{C} P^{2}$ An90, Theorem 1.3(b)].

Regarding Riemannian manifolds with almost nonnegative Ricci curvature, the following is known.

Theorem 1.8. Given $n \in \mathbb{Z}^{+}$, there are $\varepsilon=\varepsilon(n)>0$ and $J=$ $J(n)<\infty$ with the following property. Suppose that $(M, g)$ is a closed 
connected Riemannian $n$-manifold with $\operatorname{diam}(M) \leq 1$ and Ric $_{M} \geq$ $-\varepsilon$.

(1) Then $\pi_{1}(M)$ has a nilpotent subgroup (of index at most $J$ ) with nilpotency rank at most $n$ KW11. (This was also proved in BGT12 without the index bound.)

(2) If $M$ is spin and $n$ is divisible by four then $|\hat{A}(M)| \leq 2^{\frac{n}{2}-1}$ Ga83, Gr82, p. 86].

Theorem 1.7 refines Theorem 1.8(1) in the noncollapsed case, although the constants $I$ and $J$ may not be related.

Remark 1.9. In four dimensions, Rokhlin's theorem says that $\hat{A}(M)$ is even if $M$ is spin. Hence under the assumptions of Theorem 1.8(2), if $n=4$ and $\widehat{A}(M) \neq 0$ then $|\widehat{A}(M)|=2$. This means that after possibly reversing orientation, $M$ is spin-cobordant to the $K 3$ surface. One can ask whether Theorem 1.1 still holds without the upper Ricci curvature bound, say assuming a lower Ricci curvature bound and possibly a lower volume bound.

In a somewhat different direction, Cabezas-Rivas and Wilking answered a question from [Lo00] by showing that a spin manifold with almost nonnegative sectional curvature has vanishing $\widehat{A}$-genus [CW].

Regarding almost Ricci-flat 4-manifolds, Brendle and Kapouleas found an obstruction to perturbing an almost Ricci-flat metric to a Ricci-flat metric $[\mathrm{BK}$. In their example, the relevant limit space was a flat 4-dimensional orbifold. A gluing obstruction for a nonflat limit space was found by Biquard [Bi13].

Finally, one can ask whether Corollary 1.4(1) holds without the upper Ricci curvature bound.

1.4. Structure of the paper. In Section 2 we prove Theorem 1.1. In Section 3 we prove Theorem 1.3 and Corollary 1.4. In Section 4 we prove Theorem 1.7 .

We thank the participants of the MSRI Spring 2016 geometry program for helpful discussions, especially Olivier Biquard, Ronan Conlon and Jeff Viaclovsky. We also thank Jeff for comments on an earlier version of the paper. We thank the referee for pointing out BGR07.

\section{Proof of Theorem 1.1}

Arguing by contradiction, if Theorem 1.1 is not true then there is a sequence $\left\{\left(M_{i}, g_{i}\right)\right\}_{i=1}^{\infty}$ of closed connected Riemannian spin 4manifolds with

- $\widehat{A}\left(M_{i}\right) \neq 0$, 
- $\operatorname{vol}\left(M_{i}\right) \geq v$,

- $\operatorname{diam}\left(M_{i}\right) \leq 1$,

- $\operatorname{Ric}_{M_{i}} \leq \Upsilon g_{i}$ and

- $S_{M_{i}} \geq-\frac{1}{i}$, but

- $M_{i}$ is not diffeomorphic to a $K 3$ surface.

2.1. Harmonic spinors on $M_{i}$. By assumption, the second StiefelWhitney class of $M_{i}$ vanishes in $H^{2}\left(M_{i} ; \mathbb{Z}_{2}\right)$. After reversing orientation if necessary, we can assume that $\widehat{A}\left(M_{i}\right)>0$. We can then choose a spin structure on $M_{i}$ that is compatible with this orientation. The Atiyah-Singer index theorem implies that $M_{i}$ has a nonzero harmonic spinor $\phi_{i}$ of positive chirality. By rescaling, we can assume that $\left\|\phi_{i}\right\|_{2}=1$. Letting $D$ denote the Dirac operator, the Lichnerowicz formula gives

$$
0=\int_{M_{i}}\left|D \phi_{i}\right|^{2} \operatorname{dvol}_{M_{i}}=\int_{M_{i}}\left(\left|\nabla \phi_{i}\right|^{2}+\frac{S_{M_{i}}}{4}\left|\phi_{i}\right|^{2}\right) \operatorname{dvol}_{M_{i}} .
$$

Hence

$$
\int_{M_{i}}\left|\nabla \phi_{i}\right|^{2} \operatorname{dvol}_{M_{i}} \leq \frac{1}{4 i}
$$

As

$$
\nabla\left|\phi_{i}\right|=\nabla \sqrt{\left\langle\phi_{i}, \phi_{i}\right\rangle}=\frac{\left\langle\nabla \phi_{i}, \phi_{i}\right\rangle+\left\langle\phi_{i}, \nabla \phi_{i}\right\rangle}{2\left|\phi_{i}\right|}
$$

away from the zero-locus of $\phi_{i}$, we have

$$
|\nabla| \phi_{i}|| \leq\left|\nabla \phi_{i}\right|
$$

and so

$$
\int_{M_{i}}|\nabla| \phi_{i}||^{2} \operatorname{dvol}_{M_{i}} \leq \frac{1}{4 i}
$$

Let $\lambda_{i}>0$ denote the Poincaré constant of $\left(M_{i}, g_{i}\right)$, so that

$$
\int_{M_{i}}|\nabla F|^{2} \mathrm{dvol}_{M_{i}} \geq \lambda_{i} \int_{M_{i}}(F-\bar{F})^{2} \mathrm{dvol}_{M_{i}}
$$

for all $F \in H^{1}\left(M_{i}\right)$, where $\bar{F}$ denotes the average value $\bar{F}=\frac{1}{\operatorname{vol}\left(M_{i}\right)} \int_{M_{i}} F \operatorname{dvol}_{M_{i}}$. Then

$$
\int_{M_{i}}\left(\left|\phi_{i}\right|-\overline{\left|\phi_{i}\right|}\right)^{2} \mathrm{dvol}_{M_{i}} \leq \frac{1}{4 i \lambda_{i}}
$$

Lemma 2.8. If $V_{i}$ is open in $M_{i}$ then

$$
\int_{V_{i}}\left|\phi_{i}\right|^{2} d v o l_{M_{i}} \leq\left(\frac{1}{\sqrt{4 i \lambda_{i}}}+\sqrt{\frac{\operatorname{vol}\left(V_{i}\right)}{\operatorname{vol}\left(M_{i}\right)}}\right)^{2} .
$$


Proof. We work more generally with a measure space $(M, d \mu)$ having finite mass, a measurable subset $V \subset M$ and a function $f \in L^{2}(M, d \mu)$ with $\|f\|_{L^{2}(M)}=1$. By the Cauchy-Schwarz inequality, $|\bar{f}| \leq \frac{1}{\sqrt{\mu(M)}}$. Then

$$
\|f\|_{L^{2}(V)} \leq\|f-\bar{f}\|_{L^{2}(V)}+\|\bar{f}\|_{L^{2}(V)} \leq\|f-\bar{f}\|_{L^{2}(M)}+\sqrt{\frac{\mu(V)}{\mu(M)}}
$$

from which the lemma follows.

2.2. Parallel spinors on $X$. From the scalar curvature condition and the upper bound on Ricci curvature, for large $i$ we have $\operatorname{Ric}_{M_{i}} \geq$ $-10 \Upsilon g_{i}$. Then from [CN15, Theorem 1.13], there is a uniform upper bound on $\int_{M_{i}}\left|\mathrm{Rm}_{M_{i}}\right|^{2} \mathrm{dvol}_{M_{i}}$. By An90, Theorem 2.6 and Pf. of Main Lemma 2.2], after passing to a subsequence we can assume that $\lim _{i \rightarrow \infty}\left(M_{i}, g_{i}\right)=\left(X, g_{X}\right)$ in the Gromov-Hausdorff topology, where

- $X$ is a four dimensional compact orbifold with finitely many isolated orbifold singularities,

- $g_{X}$ is a continuous orbifold Riemannian metric on $X$, and

- $g_{X}$ is locally $W^{2, p}$-regular away from the singular points, for all $p<\infty$.

Let $X_{\text {reg }}$ denote the regular part of the orbifold $X$, i.e. the complement of the finitely many singular points $X_{\text {sing }}$. It is a $W^{3, p}$-manifold, for all $p<\infty$, so we can assume that it is equipped with an underlying smooth structure (although with a Riemannian metric that is $W^{2, p_{-}}$ regular in that smooth structure). Then $X_{r e g}$ is also a $C^{2, \alpha}$-manifold and $g_{X}$ is locally $C^{1, \alpha}$-regular, for all $\alpha \in(0,1)$.

For large $j$, let $U_{j}$ be the union of the $\frac{1}{j}$-balls around the singular points in $X$. Note that $X-U_{j}$ and $M_{i}$ are both smooth. For large $i$, there is a smooth map $\sigma_{j, i}:\left(X-U_{j}\right) \rightarrow M_{i}$ that is a diffeomorphism onto its image, so that $\lim _{i \rightarrow \infty} \sigma_{j, i}^{*} g_{i}=g_{X-U_{j}}$ in the $C^{1, \alpha}$-topology. In particular, $X-U_{j}$ admits a spin structure. As $H^{1}\left(X-U_{j} ; \mathbb{Z}_{2}\right)$ is finite, after passing to a subsequence of $i$ 's, we can assume that each $\phi_{j, i}$ is spin compatible. From (2.2),

$$
\int_{\sigma_{j, i}\left(X-U_{j}\right)}\left|\nabla \phi_{i}\right|^{2} \operatorname{dvol}_{M_{i}} \leq \frac{1}{4 i}
$$


From Lemma 2.8,

$$
\begin{gathered}
\int_{\sigma_{j, i}\left(X-U_{j}\right)}\left|\phi_{i}\right|^{2} \operatorname{dvol}_{M_{i}} \geq \\
1-\left(\frac{1}{\sqrt{4 i \lambda_{i}}}+\sqrt{\frac{\operatorname{vol}\left(M_{i}-\sigma_{j, i}\left(X-U_{j}\right)\right)}{\operatorname{vol}\left(M_{i}\right)}}\right)^{2} .
\end{gathered}
$$

It makes sense to compare spinor fields on two diffeomorphic Riemannian manifolds (c.f. [Lo00, p. 531-532]), so we can consider $\sigma_{j, i}^{*} \phi_{i}$, a spinor field on $X-U_{j}$. The $H^{1}$-norm on spinor fields over $X-U_{j}$ is

$$
\|\psi\|_{H^{1}\left(X-U_{j}\right)}^{2}=\int_{X-U_{j}}\left(|\psi|^{2}+|\nabla \psi|^{2}\right) \operatorname{dvol}_{X-U_{j}} .
$$

Note that the Christoffel symbols on $X-U_{j}$ are locally $C^{\alpha}$-regular and locally $W^{1, p}$-regular. From (2.11) and the normalization of $\phi_{i}$, the $H^{1}$-norms of $\left\{\sigma_{j, i}^{*} \phi_{i}\right\}_{i=1}^{\infty}$ are uniformly bounded, so we can take a subsequence that converges weakly in $H^{1}$ to some positive chirality spinor field $\psi_{j}$ on $X-U_{j}$. By Rellich compactness, after passing to a further subsequence we can assume that $\lim _{i \rightarrow \infty} \sigma_{j, i}^{*} \phi_{i}=\psi_{j}$ in $L^{2}$. In particular,

$$
\begin{aligned}
\left\|\psi_{j}\right\|_{L^{2}\left(X-U_{j}\right)}^{2} & =\lim _{i \rightarrow \infty}\left\|\sigma_{j, i}^{*} \phi_{i}\right\|_{L^{2}\left(X-U_{j}\right)}^{2}=\lim _{i \rightarrow \infty}\left\|\phi_{i}\right\|_{L^{2}\left(\sigma_{j, i}\left(X-U_{j}\right)\right)}^{2} \\
& =\lim _{i \rightarrow \infty} \int_{\sigma_{j, i}\left(X-U_{j}\right)}\left|\phi_{i}\right|^{2} \operatorname{dvol}_{M_{i}} .
\end{aligned}
$$

As norms can only decrease when taking weak limits, using (2.11) we have

$$
\begin{aligned}
\left\|\psi_{j}\right\|_{H^{1}\left(X-U_{j}\right)}^{2} & \leq \liminf _{i \rightarrow \infty}\left\|\sigma_{j, i}^{*} \phi_{i}\right\|_{H^{1}\left(X-U_{j}\right)}^{2}=\liminf _{i \rightarrow \infty}\left\|\phi_{i}\right\|_{H^{1}\left(\sigma_{j, i}\left(X-U_{j}\right)\right)}^{2} \\
& =\liminf _{i \rightarrow \infty} \int_{\sigma_{j, i}\left(X-U_{j}\right)}\left(\left|\nabla \phi_{i}\right|^{2}+\left|\phi_{i}\right|^{2}\right) \mathrm{dvol}_{M_{i}} \\
& =\lim _{i \rightarrow \infty} \int_{\sigma_{j, i}\left(X-U_{j}\right)}\left|\phi_{i}\right|^{2} \mathrm{dvol}_{M_{i}} .
\end{aligned}
$$

Thus $\left\|\psi_{j}\right\|_{H^{1}\left(X-U_{j}\right)}=\left\|\psi_{j}\right\|_{L^{2}\left(X-U_{j}\right)}$, so $\nabla \psi_{j}$ vanishes weakly.

There is a uniform positive lower bound on $\lambda_{i}$ in terms of the upper diameter bound and the lower Ricci bound; see [BQ00 and references therein. We have convergence $\lim _{i \rightarrow \infty}\left(M_{i}, g_{M_{i}}, \operatorname{dvol}_{M_{i}}\right) \rightarrow$ 
$\left(X, g_{X}, \mathrm{dvol}_{X}\right)$ in the measured Gromov-Hausdorff topology. Then using (2.12), we find

$$
1-\frac{\operatorname{vol}\left(U_{j}\right)}{\operatorname{vol}(X)} \leq\left\|\psi_{j}\right\|_{L^{2}\left(X-U_{j}\right)}^{2} \leq 1
$$

The preceding construction of $\psi_{j}$ was for a fixed but sufficiently large $j$. We can take $j \rightarrow \infty$, and apply a diagonal argument in $j$ and $i$, to obtain a weakly parallel positive chirality spinor field $\psi_{\infty}$ on the spin manifold $X_{r e g}$, with $\left\|\psi_{\infty}\right\|_{L^{2}\left(X_{r e g}\right)}=1$.

In a coordinate chart and using an orthonormal frame $\left\{e_{a}\right\}_{a=1}^{4}$, the fact that $\psi_{\infty}$ is weakly parallel means that

$$
\partial_{k} \psi_{\infty}=-\frac{1}{8} \sum_{a, b=1}^{4} \Gamma_{a b k}\left[\gamma^{a}, \gamma^{b}\right] \psi_{\infty}
$$

holds weakly, where $\left\{\gamma^{a}\right\}_{a=1}^{4}$ are the Dirac matrices. We know that $\psi_{\infty}$ is $W^{1,2}$-regular. As $\Gamma$ is $W^{1, p}$-regular for all $p<\infty$, using the Sobolev embedding theorem and bootstrapping, one finds that $\psi_{\infty}$ is locally $W^{2, p}$-regular for all $p<\infty$. In particular, $\psi_{\infty}$ is locally $C^{1, \alpha}$-regular for all $\alpha \in(0,1)$. Hence $\psi_{\infty}$ satisfies (2.17) in the classical sense. Then for any smooth vector field $V$ on $X_{\text {reg }}$, we have

$$
V\left\langle\psi_{\infty}, \psi_{\infty}\right\rangle=\left\langle\nabla_{V} \psi_{\infty}, \psi_{\infty}\right\rangle+\left\langle\psi_{\infty}, \nabla_{V} \psi_{\infty}\right\rangle=0
$$

showing that $\left|\psi_{\infty}\right|$ is a (nonzero) constant.

Since $\psi_{\infty}$ is parallel and locally $W^{2, p}$-regular, for smooth vector fields $V$ and $W$ on $X_{\text {reg }}$ we have

$$
0=\nabla_{V} \nabla_{W} \psi_{\infty}-\nabla_{W} \nabla_{V} \psi_{\infty}-\nabla_{[V, W]} \psi_{\infty}=R(V, W) \psi_{\infty}
$$

in $L_{l o c}^{p}$, for all $p<\infty$. Along with the nowhere-vanishing of $\psi_{\infty}$, this implies algebraically that $\operatorname{Ric}_{X}=0$ [BHMMM15, Corollary 2.8].

We recall that the $W^{2, p}$-regularity of $g_{X}$ around $x \in X_{\text {reg }}$ is derived using coordinates that are constructed by starting with harmonic coordinates around points $m_{i} \in M_{i}$, with $\lim _{i \rightarrow \infty} m_{i}=x$ and passing to the limit [An90, Pf. of Main Lemma 2.2]. Hence the formula for Ric in harmonic coordinates An90, (2.7)] still holds weakly in the coordinates around $x \in X$. By elliptic regularity, the vanishing of $\operatorname{Ric}_{X}$ implies that $g_{X}$ is smooth on $X_{r e g}$, relative to the smooth structure defined by these coordinates. Being parallel, $\psi_{\infty}$ is also smooth.

From [BGR07, Theorem 1], the spin structure on $X_{\text {reg }}$ extends to an orbifold spin structure on $X$. By removable singularity results for Einstein metrics, $g_{X}$ is a smooth Ricci-flat orbifold Riemannian metric on $X$; c.f. [BKN89, §5]. 
Given $p \in X_{\text {sing }}$, let $\left(U, q, \Gamma_{p}\right)$ be an orbifold chart for a neighborhood of $p$. Here $U$ is a ball in $\mathbb{R}^{4}$ around the origin $q$, and $\Gamma_{p}$ is the local group of $p$. The lift of $\psi_{\infty}$ is a parallel spinor field $\hat{\psi}_{\infty}$ on $U-q$. Since $U-q$ is simply connected, $\hat{\psi}_{\infty}$ has a unique extension to a smooth parallel spinor field over $U$. Hence $\psi_{\infty}$ extends uniquely to a nonzero positive chirality parallel spinor field on $X$. Given $x \in X_{\text {reg }}$, let $\gamma$ be a special loop at $x$ in the sense of [KL14, Chapter 2.2]. Identifying the oriented isometry group of $T_{x} X$ with $S O(4)$, from the decomposition $\operatorname{Spin}(4)=S U(2) \times S U(2)$ it follows that the holonomies around such $\gamma$ 's lie in one of the $S U(2)$-factors. (As the parallel spinor field has positive chirality, our conventions are that the $S U(2)$-factor is the second factor.) That is, $X$ acquires the structure of a hyperKähler orbifold. In particular, if $p \in X_{\text {sing }}$ then we can take the orbifold chart $\left(U, q, \Gamma_{p}\right)$ to have $U$ an open ball in $\mathbb{C}^{2}$ with origin $q$ and $\Gamma_{p}$ a finite subgroup of $S U(2)$ that acts freely on $T_{q}^{1} U \cong S^{3}$.

2.3. ALE blowups. If $p \in X_{\text {sing }}$ then there are points $p_{i} \in M_{i}$ so that $\lim _{i \rightarrow \infty}\left(M_{i}, p_{i}\right)=(X, p)$ in the pointed Gromov-Hausdorff topology. After passing to a subsequence, from [AC91, Remark 3.1] and [Ba90a, Proposition 2] there is an appropriate sequence $\left\{\delta_{i}\right\}_{i=1}^{\infty}$ of positive numbers with $\lim _{i \rightarrow \infty} \delta_{i}=0$ so that $\lim _{i \rightarrow \infty}\left(M_{i}, \frac{1}{\delta_{i}^{2}} g_{M_{i}}, p_{i}\right)=\left(Y, g_{Y}, y_{0}\right)$ in the pointed Gromov-Hausdorff topology, where $Y$ is an nonflat Ricciflat ALE orbifold with finitely many orbifold singular points. The decay rate of $Y$ is order- 4 in the terminology of [BKN89]; see [Ba90a, Proposition 2] and [BKN89, Theorem 1.5]. For any small $\epsilon>0$, the complement $C_{\epsilon}$ of the $\epsilon$-neighborhood of $Y_{\text {sing }}$ embeds in $M_{i}$ for large $i$. In particular, $C_{\epsilon}$ admits a spin structure. Since $H^{1}\left(C_{\epsilon} ; \mathbb{Z}_{2}\right)$ is finite, after passing to a subsequence we can assume that the embeddings are spin compatible. Taking $\epsilon$ going to zero, $Y_{\text {reg }}$ acquires a spin structure. From [BGR07, Theorem 1], the orbifold $Y$ is a spin orbifold. Its tangent cone at infinity is $\mathbb{C}^{2} / \Gamma_{p}$.

Lemma 2.20. $Y$ has a nonzero parallel spinor field.

Proof. The proof is essentially the same as that of Na90, Corollary 3.4], which treats the case when $Y$ is a manifold, with only minor changes. To make this clear, we outline the steps of the proof. From the existence of the positive chirality parallel spinor $\psi_{\infty}$ in a neighborhood of $p \in X$, the action of $\Gamma_{p}$ on $S^{3}$ is a right action in the sense of [Na90, p. 390]. As in Witten's proof of the positive mass theorem, one constructs a positive chirality spinor field $\eta_{0}$ on $Y$ that is asymptotically parallel at infinity, with norm approaching one. Then one puts $\eta=\eta_{0}-D G D \eta$, where $D$ is the Dirac operator and $G$ is the inverse of 
the (invertible) operator $D^{2}$, when considered as an operator between appropriate weighted function spaces. The spinor field $\eta$ is harmonic and has the same asymptotics as $\eta_{0}$. This linear analysis extends with only trivial change to the orbifold case.

Integration by parts, and the fact that $Y$ is scalar-flat, give

$$
m=\text { const. } \int_{Y}|\nabla \eta|^{2} \operatorname{dvol}_{Y},
$$

where $m$ is the ADM mass, as defined using integration over large distance spheres in $Y$. The order- 4 decay rate of $Y$ implies that $m=$ 0 . Hence the positive chirality spinor field $\eta$ is parallel.

Thus $Y$ is also a hyperKähler orbifold.

2.4. Bubble tree. We can repeat the blowup analysis at a point of $Y_{\text {sing }}$, using the fact that $Y$ has a nonzero parallel spinor field of positive chirality. The result is that we get a bubble tree $T$, as in AC91, Ba90a. (The papers Ba90a, Ba90b treat the case when the initial manifold is Einstein, while AC91 treats the more general case of bounded Ricci curvature.) This is a finite directed rooted tree, with a connected orbifold associated to each vertex. The orbifold associated to the root vertex is $X$. The orbifolds associated to the other vertices $v$ are Ricci-flat ALE orbifolds $W_{v}$. The edges of $T$ point inward toward the root vertex. Given a vertex $v \in T$, the edges with terminus $v$ are in bijective correspondence with $W_{v, \text { sing }}$. The initial vertex $v^{\prime}$ of such an edge $e$ is the result of the blowup analysis at the corresponding

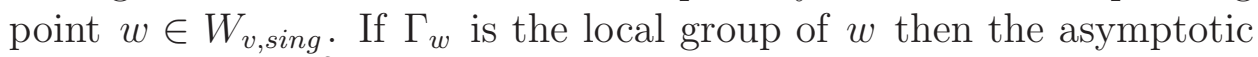
cone of $W_{v^{\prime}}$ is $\mathbb{C}^{2} / \Gamma_{w}$. The finiteness of $T$ comes from the uniform upper bound on $\int_{M_{i}}\left|\mathrm{Rm}_{M_{i}}\right|^{2} \mathrm{dvol}_{M_{i}}$, since each blowup orbifold has a definite amount of $\int|\mathrm{Rm}|^{2} \mathrm{dvol}$.

Given $k \geq 0$, let $T_{k}$ be the vertices of distance $k$ from the root vertex. Let $N$ be the largest $k$ for which $T_{k} \neq \emptyset$, which we will call the height of $T$. The orbifolds associated to vertices in $T_{N}$ are manifolds.

From $T$ we can construct a compact smooth manifold $M_{T}$ that is diffeomorphic to $M_{i}$, for large $i$. To describe $M_{T}$, first consider the case when $N=0$. Then $X$ is a smooth manifold and $M_{T}=X$. If $N=1$ then $T$ consists of the root vertex along with vertices in $T_{1}$. The only edges in $T$ join vertices in $T_{1}$ to the root vertex. Given $x \in X_{\text {sing }}$, a small neighborhood $O_{x}$ of $x$ is orbifold-diffeomorphic to a finite cone over the space form $S^{3} / \Gamma_{x}$. If $v^{\prime} \in T_{1}$ is the initial vertex of the edge corresponding to $x$ then its associated orbifold $W_{v^{\prime}}$ has asymptotic 
cone $\mathbb{C}^{2} / \Gamma_{x}$. Let $P_{x}$ be a truncation of $W_{v^{\prime}}$ whose boundary is a copy of $S^{3} / \Gamma_{x}$ at large distance. We remove $O_{x}$ from $X$ and glue in a rescaled copy of $P_{x}$. Doing this for all $x \in X_{\text {sing }}$ gives $M_{T}$. Given the combinatorics of the matchings, the gluing process is unique up to isotopy, because of the existence of the small-scale asymptotics near $x$ and the large-scale asymptotics of $W_{v^{\prime}}$.

For $N>1$, we do the same gluing procedure inductively. That is, given a vertex $v \in T_{N-1}$, if there are no edges terminating at $v$ then the orbifold associated to $v$ is a manifold and we leave it alone. If there are edges terminating at $v$ then the orbifold associated to $v$ has singular points. We remove neighborhoods of the singular points and glue in truncated manifolds associated to the vertices $v^{\prime} \in T_{N}$ that are joined to $v$ in $T$. Doing this for all $v \in T_{N-1}$, the result is a tree of height $N-1$ for which the orbifolds associated to the vertices of distance $N-1$ from the root vertex are all noncompact manifolds. Then we iterate downward in the height of the tree, until we finish with $M_{T}$.

From the uniqueness of the gluing procedure, up to isotopy, if we know the orbifolds associated to the vertices of $T$ and the combinatorics of the matchings then we can uniquely determine the diffeomorphism type of $M_{T}$.

2.5. End of the proof. In the previous subsection, we did not make reference to the hyperKähler structures. In our situation, all of the orbifolds associated to the vertices of $T$ carry hyperKähler structures.

Lemma 2.22. For large $i$, the manifold $M_{i}$ is diffeomorphic to a hyperKähler manifold.

Proof. Consider the height $N$ of the bubble tree $T$. If $N=0$ then $M_{i}$ is diffeomorphic to the hyperKähler manifold $X$. If $N>0$ then the orbifolds associated to the vertices of $T$ are hyperKähler ALE orbifolds. Those associated to vertices in $T_{N}$ are hyperKähler ALE manifolds.

For a hyperKähler ALE manifold associated to a vertex in $T_{N}$, if its asymptotic cone is $\mathbb{C}^{2} / \Gamma$ then the manifold is deformation equivalent to the minimal resolution of $\mathbb{C}^{2} / \Gamma$, through hyperKähler ALE manifolds with asymptotic cone $\mathbb{C}^{2} / \Gamma$ [Jo00, Theorem 7.2.3], Kr89]. Consequently, for our gluing purposes, we can assume that the hyperKähler structure on the ALE manifold is exactly that of the minimal resolution.

Consider first the case $N=1$. Using the compatible trivializations of the positive chirality spinor bundles, in a neighborhood of a singular point of $X$ and at the infinity of the corresponding ALE manifold, there 
is no ambiguity in the matchings. We see that $M_{T}$ is the minimal resolution of the hyperKähler orbifold $X$. As $X$ is hyperKähler, it has a trivial canonical bundle. The minimal resolution of an orbifold of complex dimension two is a crepant resolution, i.e. the minimal resolution of $X$ also has a trivial canonical bundle. Hence it admits a hyperKähler structure.

If $N>1$ then let $v$ be a vertex in $T_{N-1}$, with associated hyperKähler ALE orbifold $W_{v}$. If there are no edges terminating at $v$ then $W_{v}$ is a hyperKähler ALE manifold. As before, we deform it to a minimal resolution of a $\mathbb{C}^{2} / \Gamma$. If there are edges terminating at $v$ then $W_{v}$ is a hyperKähler ALE orbifold with singular points. From Ba90a, Theorem 4], after we perform the gluing procedure around the orbifold singular points of $W_{v}$, the result has the structure of a hyperKähler ALE manifold. We again deform it to a minimal resolution of a $\mathbb{C}^{2} / \Gamma$.

Doing this for all $v \in T_{N-1}$, we have reduced to a tree of height $N-1$. The lemma follows from downward iteration.

Lemma 2.22 says that for large $i$, the manifold $M_{i}$ is diffeomorphic to a compact hyperKähler 4-manifold. Such a hyperKähler manifold is a 4 -torus or a $K 3$ surface [BHPV04, Chapter 6]. Since $\widehat{A}\left(T^{4}\right)=$ 0 , in either case we obtain a contradiction to the assumptions of the argument.

Remark 2.23. From the gluing procedure, one obtains geometric approximations for the $M_{i}$ 's, for large $i$. It seems possible that one could perturb the geometric approximation, as in [BM11, Theorem 2.5], in order to find a Ricci-flat metric on $M_{i}$ that is biLipschitz close to $g_{i}$.

Remark 2.24. The direct higher dimensional analog of Theorem 1.1 would be to ask whether for any $n \in \mathbb{Z}^{+}, v>0$ and $\Lambda, \Upsilon<\infty$, there is some $\epsilon=\epsilon(n, v, \Lambda, \Upsilon)>0$ so that if $M$ is a closed connected spin Riemannian $n$-manifold with $\widehat{A}(M) \neq 0, \operatorname{vol}(M) \geq v, \operatorname{diam}(M) \leq 1$, $\int_{M}|\operatorname{Rm}|^{\frac{n}{2}} \mathrm{dvol}_{M} \leq \Lambda, \operatorname{Ric}_{M} \leq \Upsilon g_{M}$ and $S_{M} \geq-\epsilon$, then $M$ admits a Ricci-flat metric of special holonomy.

The discussions of Subsections 2.3,2.4 go through without change to produce a limit orbifold $X$ with special holonomy, blowup Ricci-flat ALE orbifolds with special holonomy and a bubble tree. However, if $n>4$ then there are more possibilities for the holonomies and hence more possibilities for the orbifolds associated to the vertices of the bubble tree. Rather than trying to classify the possibilities, it is conceivable that one could perform a gluing construction, as mentioned in Remark 2.23, in order to directly construct Ricci-flat metrics of special holonomy. 


\section{Proofs of Theorem 1.3 and Corollary 1.4}

We first prove Theorem 1.3(1). Arguing by contradiction, if it is not true then for some $n \in \mathbb{Z}^{+}, v>0$ and $\Lambda, \Upsilon<\infty$, there is a sequence $\left\{\left(M_{i}, g_{i}\right)\right\}_{i=1}^{\infty}$ of closed connected $n$-dimensional Riemannian manifolds with

- $\int_{M_{i}}\left|\mathrm{Rm}_{M_{i}}\right|^{\frac{n}{2}} \mathrm{dvol}_{M_{i}} \leq \Lambda$,

- $\operatorname{vol}\left(M_{i}\right) \geq v$,

- $\operatorname{diam}\left(M_{i}\right) \leq 1$,

- $-\frac{1}{i} g_{i} \leq \operatorname{Ric}_{M_{i}} \leq \Upsilon g_{i}$ and

- $\left|\pi_{1}\left(M_{i}\right)\right| \geq i$, but

- $M_{i}$ does not admit a $W^{2, p}$-regular Riemannian metric $h_{i}$ with nonnegative measurable Ricci curvature for which the universal cover $\left(\tilde{M}_{i}, \tilde{h}_{i}\right)$ isometrically splits off an $\mathbb{R}$-factor.

By [An90, Theorem 2.6], after passing to a subsequence we can assume that $\lim _{i \rightarrow \infty}\left(M_{i}, g_{i}\right)=\left(X, g_{X}\right)$ in the Gromov-Hausdorff topology, where

- $X$ is an $n$-dimensional compact orbifold with finitely many isolated orbifold singularities,

- $g_{X}$ is a continuous orbifold Riemannian metric on $X$, and

- $g_{X}$ is locally $C^{1, \alpha}$-regular away from the singular points, for all $\alpha \in(0,1)$.

As before, $g_{X}$ is locally $W^{2, p}$-regular on $X_{\text {reg }}$, for all $p<\infty$. In particular, $g_{X}$ has a locally- $L^{p}$ Ricci tensor.

Given $x \in X$, there are points $p_{i} \in M_{i}$ so that $\lim _{i \rightarrow \infty}\left(M_{i}, p_{i}\right)=$ $(X, x)$ in the pointed Gromov-Hausdorff topology. Put $\Gamma_{i}=\pi_{1}\left(M_{i}, p_{i}\right)$, let $\pi_{i}: \tilde{M}_{i} \rightarrow M_{i}$ be the universal cover of $M_{i}$ equipped with the pullback metric $g_{\tilde{M}_{i}}$, and pick $\tilde{p}_{i} \in \pi_{i}^{-1}\left(p_{i}\right)$. After passing to a subsequence, we can assume that $\lim _{i \rightarrow \infty}\left(\tilde{M}_{i}, \tilde{p}_{i}, \Gamma_{i}\right)=(Y, \tilde{p}, \Gamma)$ in the equivariant pointed Gromov-Hausdorff topology of [FY92, §3], where $(Y, \tilde{p})$ is a pointed length space on which $\Gamma$ acts by isometries, with quotient $X$.

Since $\left|\pi_{1}\left(M_{i}\right)\right| \geq i$, we know that $\operatorname{vol}\left(\tilde{M}_{i}\right) \geq i v$, so $\lim _{i \rightarrow \infty} \operatorname{vol}\left(\tilde{M}_{i}\right)=$ $\infty$. Using the fact that $\operatorname{Ric}_{M_{i}} \geq-1$, volume comparison implies that $\lim _{i \rightarrow \infty} \operatorname{diam}\left(\tilde{M}_{i}\right)=\infty$. Hence $\operatorname{diam} Y=\infty$ and $Y$ is noncompact. We have $\operatorname{Ric}_{M_{i}} \geq-\frac{1}{i}$, so the Cheeger-Colding almost splitting theorem [CC96, Theorem 6.64] holds on $Y$. As the action of $\Gamma$ on $Y$ is cocompact, the Cheeger-Gromoll argument [CG72, §9] implies that $Y$ is isometric to $\mathbb{R}^{m} \times Z$, where $1 \leq m \leq n$ and $Z$ is a compact length space. 
Lemma 3.1. Given $r>0$, there is a upper bound on the integral of $\left|R m_{\tilde{M}_{i}}\right|^{\frac{n}{2}}$ over $r$-balls in $\tilde{M}_{i}$, uniform in $i$.

Proof. Since $\operatorname{Ric}_{M_{i}} \geq-\frac{1}{i} g_{i}$, Bishop-Gromov relative volume comparison gives an explicit $c=c(n, v, r)>0$ such that for any $m_{i} \in M_{i}$, we have $\operatorname{vol}\left(B_{r}\left(m_{i}\right)\right) \geq c$. Let $\tilde{m}_{i}$ be a preimage of $m_{i}$ in $\tilde{M}_{i}$. Absolute volume comparison gives an explicit $c^{\prime}=c^{\prime}(n, r)<\infty$ such that $\operatorname{vol}\left(B_{10 r}\left(\tilde{m}_{i}\right)\right) \leq c^{\prime}$. Suppose now that some $m_{i}^{\prime} \in B_{r}\left(m_{i}\right)$ has $N$ preimages in $B_{r}\left(\tilde{m}_{i}\right)$. Then any $m_{i}^{\prime \prime} \in B_{r}\left(m_{i}\right)$ has at least $N$ preimages in $B_{10 r}\left(\tilde{m}_{i}\right)$. Thus

$$
N \leq \frac{\operatorname{vol}\left(B_{10 r}\left(\tilde{m}_{i}\right)\right)}{\operatorname{vol}\left(B_{r}\left(m_{i}\right)\right)} \leq \frac{c^{\prime}}{c}
$$

Hence

$$
\int_{B_{r}\left(\tilde{m}_{i}\right)}\left|\operatorname{Rm}_{\tilde{M}_{i}}\right|^{\frac{n}{2}} \operatorname{dvol}_{\tilde{M}_{i}} \leq \frac{c^{\prime}}{c} \int_{M_{i}}\left|\operatorname{Rm}_{M_{i}}\right|^{\frac{n}{2}} \operatorname{dvol}_{M_{i}} \leq \frac{c^{\prime}}{c} \Lambda .
$$

This proves the lemma.

Using Lemma 3.1, we can apply An90 to conclude that $Y$ is an $n$-dimensional orbifold with a discrete set of isolated orbifold singular points, and a nonnegative measurable Ricci tensor. From the splitting $Y \cong \mathbb{R}^{m} \times Z$, the set of singular points in $Y$ must be empty. Then $\lim _{i \rightarrow \infty}\left(\tilde{M}_{i}, \tilde{p}_{i}\right)=(Y, \tilde{p})$, with Riemannian metrics converging in the pointed weak $W^{2, p}$-topology; c.f. An90, Remark 2.7(ii)]. In particular, $Y$ has nonnegative measurable Ricci curvature.

Since $X=Y / \Gamma$ is $n$-dimensional, it follows that $\Gamma \subset \operatorname{Iso}(Y)$ is discrete. A priori, $\Gamma$ need not act freely on $Y$. However, since the orbifold singular points of $X$ are isolated, if $\Gamma$ does not act freely on $Y$ then the points in $Y$ with nontrivial isotropy groups must be isolated. We claim that the action is free in our situation.

Lemma 3.4. The group $\Gamma$ acts freely on $Y$.

Proof. Suppose to the contrary that there is a point $q \in Y$ with a nontrivial isotropy group. Let $\gamma \in \Gamma$ be a nontrivial element that fixes $q$. Then $q$ is isolated in the fixed point set of $\gamma$. Since the action of $\gamma$ near $q$ can be linearized, there exist $r>0$ and $\delta \in(0, r / 100)$ such that $B_{s}(q)$ is a topological ball for any $s \in(0,10 r)$, and $d_{Y}(\gamma(y), y)>\delta$ for any $y \in Y$ with $r / 2<d_{Y}(q, y)<2 r$.

Let $\gamma_{i} \in \Gamma_{i}$ converge to $\gamma$ and let $q_{i} \in \tilde{M}_{i}$ converge to $q$. Since $\gamma(q)=q$ we have $\lim _{i \rightarrow \infty} d_{\tilde{M}_{i}}\left(\gamma_{i}\left(q_{i}\right), q_{i}\right)=0$. Hence for large $i$,

$$
B_{r-\frac{\delta}{100}}\left(q_{i}\right) \subset \gamma_{i}\left(B_{r}\left(q_{i}\right)\right) \subset B_{r+\frac{\delta}{100}}\left(q_{i}\right) .
$$


Also, $d_{\tilde{M}_{i}}\left(\gamma_{i}\left(\tilde{m}_{i}\right), \tilde{m}_{i}\right)>\delta / 2$ for any $\tilde{m}_{i} \in \tilde{M}_{i}$ with $r / 2<d_{\tilde{M}_{i}}\left(\tilde{m}_{i}, q_{i}\right)<$ $2 r$. Since $\lim _{i \rightarrow \infty}\left(\tilde{M}_{i}, q_{i}\right)=(Y, q)$ in the pointed $C^{1, \alpha}$-topology, we can find a closed topological 4-disk $D_{i} \subset \tilde{M}_{i}$ which is $\varepsilon_{i}$-Hausdorff close to $B_{r}\left(q_{i}\right)$ with $\varepsilon_{i} \rightarrow 0$. Its image $\gamma_{i}\left(D_{i}\right)$ is $\varepsilon_{i}$-Hausdorff close to $\gamma_{i}\left(B_{r}\left(q_{i}\right)\right)$. Then for large $i$, equation (3.5) implies that $\gamma_{i}\left(D_{i}\right)$ is $\frac{\delta}{50}$-Hausdorff close to $B_{r}\left(q_{i}\right)$. Using the fact that $B_{2 r}\left(q_{i}\right)$ is $C^{1, \alpha}$-close to $B_{2 r}(q) \subset Y$, by slightly squeezing $B_{2 r}\left(q_{i}\right)$ inward we can find a continuous map $\phi_{i}: B_{2 r}\left(q_{i}\right) \rightarrow \tilde{M}_{i}$ such that

- $\phi_{i}$ acts as the identity on $B_{r-\delta / 10}\left(q_{i}\right)$,

- $\phi_{i}$ sends $B_{r+\delta / 20}\left(q_{i}\right)$ into $B_{r-\delta / 20}\left(q_{i}\right)$, and

- $d_{\tilde{M}_{i}}\left(\phi_{i}\left(\tilde{m}_{i}\right), \tilde{m}_{i}\right)<\frac{\delta}{5}$ for all $\tilde{m}_{i} \in B_{2 r}\left(q_{i}\right)$.

Then $\phi_{i}\left(\gamma_{i}\left(D_{i}\right)\right) \subset D_{i}$.

The map $\phi_{i} \circ \gamma_{i}: D_{i} \rightarrow D_{i}$ is continuous. We wish to show that for large $i$, it has no fixed points. If $\tilde{m}_{i} \in B_{r-\delta / 5}\left(q_{i}\right)$ then for large $i$, we have $\gamma_{i}\left(\tilde{m}_{i}\right) \in B_{r-\delta / 10}\left(q_{i}\right)$ and so $\left(\phi_{i} \circ \gamma_{i}\right)\left(\tilde{m}_{i}\right)=\gamma_{i}\left(\tilde{m}_{i}\right) \neq \tilde{m}_{i}$. If $\tilde{m}_{i} \in D_{i}$ but $\tilde{m}_{i} \notin B_{r-\delta / 5}\left(q_{i}\right)$ then

$$
\begin{aligned}
& d_{\tilde{M}_{i}}\left(\left(\phi_{i} \circ \gamma_{i}\right)\left(\tilde{m}_{i}\right), \tilde{m}_{i}\right) \geq \\
& d_{\tilde{M}_{i}}\left(\gamma_{i}\left(\tilde{m}_{i}\right), \tilde{m}_{i}\right)-d_{\tilde{M}_{i}}\left(\left(\phi_{i} \circ \gamma_{i}\right)\left(\tilde{m}_{i}\right), \gamma_{i}\left(\tilde{m}_{i}\right)\right)>\frac{3}{10} \delta .
\end{aligned}
$$

Hence $\phi_{i} \circ \gamma_{i}$ has no fixed points in $D_{i}$. This contradicts the Brouwer fixed point theorem, so $\Gamma$ must act freely on $Y$.

We now know that $X$ is a $W^{3, p}$-manifold. Therefore, from convergence theory, $M_{i}$ is $W^{3, p}$-diffeomorphic to $X$ for all large $i$. Pulling back the metric on $X$ to $M_{i}$ gives a metric $h_{i}$ that contradicts our assumptions. This proves Theorem 1.3(1).

To prove part (2) of the theorem, we replace the upper Ricci curvature bound in the contradiction argument, by the assumption that

- $\left|\operatorname{Ric}_{M_{i}}\right| \leq \frac{1}{i}$.

We construct the orbifold $X$ as before. Because of the Ricci pinching of $M_{i}$, the metric $g_{X}$ has vanishing measurable Ricci tensor away from the singular points. Then $g_{X}$ is smooth and Ricci-flat away from the singular points. By removable singularity results for Einstein metrics, $g_{X}$ is a smooth Ricci-flat orbifold Riemannian metric on $X$; c.f. BKN89, $\S 5]$. The rest of the proof proceeds as before.

We now prove part (1) of Corollary 1.4 From CN15, Theorem 1.13], for any $v>0$ there is a constant $\Lambda=\Lambda(v)>0$ so that $\operatorname{vol}(M) \geq v$, $\operatorname{diam}(M) \leq 1$ and $\left|\operatorname{Ric}_{M}\right| \leq 3$ imply that $\int_{M}|\operatorname{Rm}|^{2} \operatorname{dvol}_{M} \leq \Lambda$. Without loss of generality, we can assume that the constant $\epsilon$ in Theorem 1.3 is less than 3. From the proof of Theorem 1.3(1), we obtain a 
$W^{2, p}$-regular metric $h$ on $M$ with nonnegative measurable Ricci curvature for which the universal cover $(\tilde{M}, \tilde{h})$ is an isometric product $\mathbb{R}^{m} \times K$, where $1 \leq m \leq n$ and $K$ is a compact length space. Since isometries take lines to lines, the arguments of [CG72, §9] show that $\operatorname{Iso}\left(\mathbb{R}^{m} \times K\right) \cong \operatorname{Iso}\left(\mathbb{R}^{m}\right) \times \operatorname{Iso}(K)$, with the action of $\pi_{1}(M)$ on $\mathbb{R}^{m} \times K$ being diagonal. By CN12, Theorem 1.21], Iso $(K)$ is a Lie group.

Now $K$ is a compact $W^{3, p}$-manifold of dimension at most three, equipped with a $W^{2, p}$-regular Riemannian metric $g_{K}$ having nonnegative measurable Ricci tensor. Also, Iso $(K)$ is a compact Lie group $G$ that acts on $K$ by $W^{3, p}$-diffeomorphisms. We can fix an underlying smooth structure on $K$ for which $G$ acts by smooth diffeomorphisms; c.f. [Pa70, Theorem B]. (Any two such smooth structures are $G$-diffeomorphic; c.f. [Pa70, Theorem A].) We can slightly smooth $g_{K}$ to get a sequence $\left\{g_{k}\right\}_{k=1}^{\infty}$ of Riemannian metrics on $K$ with diameter uniformly bounded above, volume uniformly bounded below by a positive number, and $\operatorname{Ric}_{g_{k}} \geq-\frac{1}{k}$. Furthermore, this smoothing can be done equivariantly with respect to $G$. (For example, we could pick a smooth $G$-invariant metric on $K$ and apply the ensuing heat operator, acting on symmetric 2-tensor fields, to our $W^{2, p}$-regular Riemannian metric for short time.) From [Si12, Corollary 1.12], there is a smooth Riemannian metric on $K$ with nonnegative Ricci curvature. The construction of this metric, using Ricci flow, can be done $G$-equivariantly. Hence we obtain a smooth metric $h_{\infty}$ on $\left(\mathbb{R}^{m} \times K\right) / \pi_{1}(M)$ with nonnegative Ricci curvature. There is a $C^{\infty}$-diffeomorphism from $M$ to $\left(\mathbb{R}^{m} \times K\right) / \pi_{1}(M)$, the latter being equipped with the quotient smooth structure. Pulling back $h_{\infty}$ to $M$, part (1) of the corollary follows.

The proof of part (2) of the corollary is similar but easier, using Theorem 1.3(2). In this case, $K$ is Ricci-flat. Since it has dimension at most three, it is flat. Hence the metric $h^{\prime}$ of Theorem 1.3)(2) is flat. The corollary follows.

Remark 3.7. In the conclusions of Theorem 1.3 and Corollary 1.4, a finite cover of $M$ is diffeomorphic to a product $S^{1} \times N$.

Remark 3.8. With regard to the conclusion of Corollary 1.4(2), we can also say that the flat metric is $C^{1, \alpha}$-close to the original metric $g$.

\section{Proof of Theorem 1.7}

Arguing by contradiction, if Theorem 1.7 is not true then there is a sequence $\left\{\left(M_{i}, g_{i}\right)\right\}_{i=1}^{\infty}$ of closed connected Riemannian $n$-manifolds with

- $\operatorname{vol}\left(M_{i}\right) \geq v$, 
- $\operatorname{diam}\left(M_{i}\right) \leq 1$ and

- $\operatorname{Ric}_{M_{i}} \geq-\frac{1}{i}$, but

- $\pi_{1}\left(M_{i}\right)$ does not have an abelian subgroup (of index at most $i$ ) generated by at most $n$ elements.

By Gromov's precompactness theorem, we can assume that $\lim _{i \rightarrow \infty}\left(M_{i}, g_{i}\right)=$ $\left(X, d_{X}\right)$ in the Gromov-Hausdorff topology, where $\left(X, d_{X}\right)$ is a compact length space. The uniform lower volume bound on the $M_{i}$ 's implies that $X$ has Hausdorff dimension $n$ [CC97, Theorem 5.9].

Let $p \in X$ be a regular point, meaning that every tangent cone $T_{p} X$ is isometric to $\mathbb{R}^{n}$. The existence of regular points is guaranteed by [CC97, Theorem 2.1]. A small neighborhood of $p$ is homeomorphic to an open ball in $\mathbb{R}^{n}$ [CC97, Theorem A.1.8]. Furthermore, for any sequence $p_{i} \in M_{i}$ converging to $p$, there is some $\varepsilon>0$ such that for all large $i$, the ball $B_{\varepsilon}\left(p_{i}\right)$ is contained in a topological disk $D_{i} \subset$ $M_{i}$ [CC97, Theorem A.1.8].

In particular, for all large $i$, any loop at $p_{i}$ of length at most $\varepsilon / 2$ is contractible.

By passing to a subsequence, we can assume that this is true for all $i$.

As in Section 3, put $\Gamma_{i}=\pi_{1}\left(M_{i}, p_{i}\right)$, let $\pi_{i}: \tilde{M}_{i} \rightarrow M_{i}$ be the universal cover of $M_{i}$ equipped with the pullback metric $g_{\tilde{M}_{i}}$, and pick $\tilde{p}_{i} \in \pi_{i}^{-1}\left(p_{i}\right)$. After passing to a subsequence, we can assume that $\lim _{i \rightarrow \infty}\left(\tilde{M}_{i}, \tilde{p}_{i}, \Gamma_{i}\right)=(Y, \tilde{p}, \Gamma)$ in the equivariant pointed GromovHausdorff topology, where $\Gamma$ is a closed subgroup of $\operatorname{Iso}(Y)$ and $Y / \Gamma=$ $X$.

Given $\gamma_{i} \in \Gamma_{i}$ we will refer to $\left|\gamma_{i}\right|=d\left(\tilde{p}_{i}, \gamma_{i}\left(\tilde{p}_{i}\right)\right)$ as the norm or the length of $\gamma_{i}$. We will use the same notation for elements $\gamma$ of $\Gamma$. By (4.1), any nontrivial $\gamma_{i} \in \Gamma_{i}$ satisfies $\left|\gamma_{i}\right| \geq \varepsilon / 2$. This property passes to the limit, so

$$
\text { any nontrivial } \gamma \in \Gamma \text { satisfies }|\gamma|=d(\tilde{p}, \gamma \tilde{p}) \geq \varepsilon / 2 \text {. }
$$

The length space $Y$ satisfies the splitting theorem CC96, Theorem 6.64]. Since the action of $\Gamma$ on $X$ is cocompact, the Cheeger-Gromoll argument [CG72, §9] implies that $Y$ is isometric to $\mathbb{R}^{m} \times Z$ where $Z$ is a compact length space and $m \leq n$. Furthermore, since isometries take lines to lines, the arguments of [CG72, §9] show that $\operatorname{Iso}(Y) \cong$ Iso $\left(\mathbb{R}^{m}\right) \times \operatorname{Iso}(Z)$, with the action of $\Gamma$ on $Y=\mathbb{R}^{m} \times Z$ being diagonal. Let $\phi: \Gamma \rightarrow \operatorname{Iso}\left(\mathbb{R}^{m}\right)$ be the composition of inclusion $\Gamma \rightarrow \operatorname{Iso}(Y)$ and projection onto the first factor. Put $H=\operatorname{ker} \phi$. Then $H$ is a subgroup of the compact group $\operatorname{Iso}(Z)$. Property (4.2) and the compactness of $Z$ imply that $H$ is a discrete subgroup of $\operatorname{Iso}(Z)$, and hence is finite. Again using property (4.2), we obtain that $L=\phi(\Gamma)$ is a closed discrete 
subgroup of $\operatorname{Iso}\left(\mathbb{R}^{m}\right)$. It must be cocompact and hence it contains a free abelian subgroup of finite index and rank $m$, consisting only of translations. There is a short exact sequence

$$
1 \rightarrow H \rightarrow \Gamma \rightarrow L \rightarrow 1,
$$

where $H$ is finite, $\Gamma$ is finitely presented and $L$ is crystallographic.

Let $\left\langle\gamma_{1}, \ldots \gamma_{N_{1}} \mid w_{1}, \ldots, w_{N_{2}}\right\rangle$ be a presentation of $\Gamma$. By possibly increasing the generating set, we can assume that it contains all of the elements $\gamma$ of $\Gamma$ with $|\gamma| \leq 10$. The number of such elements is still bounded because of (4.2) and Bishop-Gromov volume comparison. Let $T$ be the maximum wordlength of the relations $w_{1}, \ldots, w_{N_{2}}$.

For large $i$, properties (4.1) and (4.2), along with the definition of equivariant pointed Gromov-Hausdorff convergence [FY92, §3], imply that for any generator $\gamma_{j}$, there is a unique $\gamma_{j}^{i} \in \Gamma_{i}$ that approximates $\gamma_{j}$ in the sense of [FY92, Definition 3.3]. In other words, all generators $\gamma_{j}$ have unique "lifts" to $\Gamma_{i}$. In particular, $\gamma_{j}^{i}$ will be uniformly $\frac{\varepsilon}{10 T}$ close to $\gamma_{j}$ on $B_{10 T}\left(\tilde{p}_{i}\right)$, in the sense of [FY92, Definition 3.3(4,5)]. Hence any relation $w_{s}\left(\gamma_{1}, \ldots, \gamma_{N_{1}}\right)=1$ remains true for the lifts, i.e. $w_{s}\left(\gamma_{1}^{i}, \ldots, \gamma_{N_{1}}^{i}\right)=1$, as otherwise one would obtain a noncontractible loop at $p_{i}$ with length at most $\varepsilon / 2$.

Thus there is a homomorphism $\rho_{i}: \Gamma \rightarrow \Gamma_{i}$ defined by $\rho_{i}\left(\gamma_{k}\right)=\gamma_{k}^{i}$. Now $\rho_{i}$ is an epimorphism, since $\pi_{1}\left(M_{i}, p_{i}\right)$ is generated by loops of length at most $2 \operatorname{diam}\left(M_{i}\right) \leq 2$, and the image of $\rho_{i}$ contains all such elements by construction. From (4.3), it follows that $\Gamma$ contains a finite-index free abelian subgroup $\Gamma^{\prime}$ of rank $m$. Call the index $c$. Then $\rho_{i}\left(\Gamma^{\prime}\right)$ is an abelian subgroup of $\Gamma_{i}$ (of index at most $c$ ) generated by at most $n$ elements. This contradicts our assumptions and proves Theorem 1.7 .

\section{REFERENCES}

[An90] M. Anderson. Convergence and rigidity of manifolds under Ricci curvature bounds. Inv. Math. 102(2):429-445, 1990.

[An92] M. Anderson. Hausdorff perturbations of Ricci-flat manifolds and the splitting theorem. Duke Math. J. 68(1):67-82, 1992.

[AC91] M. Anderson and J. Cheeger. Diffeomorphism finiteness for manifolds with Ricci curvature and $L^{n / 2}$-norm of curvature bounded. Geom. Funct. Anal. 1(3):231-252, 1991.

[BGT12] E. Breuillard, B. Green and T. Tao. The structure of approximate groups Publ. Math. Inst. Hautes Études Sci. 116:115-221, 2012.

[BQ00] D. Bakry and Z. Qian. Some new results on eigenvectors via dimension, diameter, and Ricci curvature. Adv. Math. 155(1):98-153, 2000.

[Ba90a] S. Bando. Bubbling out of Einstein manifolds. Tohoku Math. J. (2) 42(2):205-216, 1990. 
[Ba90b] S. Bando. Correction and addition: "Bubbling out of Einstein manifolds". Tohoku Math. J. (2) 42(4):587-588, 1990.

[BKN89] S. Bando, A. Kasue and H. Nakajima. On a construction of coordinates at infinity on manifolds with fast curvature decay and maximal volume growth. Inv. Math. 97(2):313-349, 1989.

[BHPV04] W. Barth, K. Hulek, C. Peters and A. Van de Ven. Compact complex surfaces. Springer-Verlag, Berlin, 2004.

[BGR07] F. Belgun, N. Ginoux and H.-B. Rademacher. A singularity theorem for twistor spinors. Annales de l'Inst. Fourier 57(4):1135-1159, 2007.

[Bi13] O. Biquard. Désingularisation de métriques d'Einstein, I. Inv. Math. 192(1): 197-252, 2013.

[BM11] O. Biquard and V. Minerbe. A Kummer construction for gravitational instantons. Comm. Math. Phys. 308(3):773-794, 2011.

[BHMMM15] J.-P. Bourguignon, O. Hijazi, J.-L. Milhorat, A. Moroianu and S. Moroianu. A spinorial approach to Riemannian and conformal geometry. EMS Monographs in Mathematics, European Mathematical Society, Zürich, 2015.

[BK] S. Brendle and N. Kapouleas. Gluing Eguchi-Hanson metrics and a question of Page. Comm. Pure and Applied Math. 70(7):1366-1401, 2017.

$[\mathrm{CW}]$ E. Cabezas-Rivas and B. Wilking. to appear.

[Ch69] J. Cheeger. Pinching theorems for a certain class of Riemannian manifolds. Amer. J. Math. 91:807-834, 1969.

[CC96] J. Cheeger and T. Colding. Lower bounds on Ricci curvature and the almost rigidity of warped products. Ann. of Math. 144(1):189-237, 1996.

[CC97] J. Cheeger and T. Colding. On the structure of spaces with Ricci curvature bounded below I. J. Differential Geom. 46(3):406-480, 1997.

[CG71] J. Cheeger and D. Gromoll. The splitting theorem for manifolds of nonnegative Ricci curvature J. Differential Geometry 6:119-128, 1971/72.

[CG72] J. Cheeger and D. Gromoll. On the structure of complete manifolds of nonnegative curvature. Ann. of Math. 96:413-443, 1972.

[CN15] J. Cheeger and A. Naber. Regularity of Einstein manifolds and the codimension 4 conjecture. Ann. of Math. 182(3):1093-1165, 2015.

[CN12] T. Colding and A. Naber. Sharp Hölder continuity of tangent cones for spaces with a lower Ricci curvature bound and applications. Ann. of Math. 176(2):1173-1229, 2012.

[FY92] K. Fukaya and T. Yamaguchi. The fundamental groups of almost nonnegatively curved manifolds. Ann. of Math. 136(2):253-333, 1992.

[Ga83] S. Gallot. Inégalités isopérimétriques, courbure de Ricci et invariants géométriques II. C. R. Acad. Sci. Paris Sér. I Math. 296(8):365-368, 1983.

[Gr78] M. Gromov. Almost flat manifolds. J. Differential Geom. 13(2):231-241, 1978.

[Gr82] M. Gromov. Volume and bounded cohomology Inst. Hautes Études Sci. Publ. Math. 56:5-99, 1982.

[Ha86] R. Hamilton. Four-manifolds with positive curvature operator. J. Differential Geom. 24(2):153-179 (1986)

[Jo00] D. Joyce. Compact manifolds with special holonomy. Oxford University Press, Oxford, 2000.

[KW11] V. Kapovitch and B. Wilking. Structure of fundamental groups of manifolds with Ricci curvature bounded below. http://arxiv.org/abs/1105.5955 
[KL14] B. Kleiner and J. Lott. Geometrization of three-dimensional orbifolds via Ricci flow. Astérisque 365:101-177, 2014.

[Kr89] P. Kronheimer. A Torelli-type theorem for gravitational instantons. J. Differential Geom. 29(3):685-697, 1989.

[Lo00] J. Lott. Â-genus and collapsing. J. Geom. Anal. 10(3):529-543, 2000.

[Na90] H. Nakajima. Self-duality of ALE Ricci-flat 4-manifolds and positive mass theorem. In Recent topics in differential and analytic geometry, volume 18 of Adv. Stud. Pure Math., pages 385-396. Academic Press, Boston, MA, 1990.

[Pa70] R. Palais. $C^{1}$ actions of compact Lie groups on compact manifolds are $C^{1}$ equivalent to $C^{\infty}$ actions. Amer. J. Math. 92:748-760, 1970.

[Si12] M. Simon. Ricci flow of non-collapsed three manifolds whose Ricci curvature is bounded from below. J. Reine Angew. Math. 662:59-94, 2012.

Vitali Kapovitch, Department of Mathematics, University of Toronto, Toronto, ON, Canada M5S 2E4,

E-mail address: vtk@math.toronto.edu

John Lott, Department of Mathematics, University of California-

Berkeley, Berkeley, CA 94720-3804,

E-mail address: lott@berkeley.edu 